\title{
High Responsivity Ultraviolet Photoconductors Based on Epitaxial ZnO Thin Films
}

\author{
Ahmed Mohammed Nahhas \\ Department of Electrical Engineering, Faculty of Engineering and Islamic Architecture, Umm Al Qura University, Makkah, Saudi Arabia \\ Email address: \\ amnahhas@uqu.du.sa \\ To cite this article: \\ Ahmed Mohammed Nahhas. High Responsivity Ultraviolet Photoconductors Based on Epitaxial ZnO Thin Films. American Journal of Nano \\ Research and Applications. Special Issue: Recent Advances of Nanomaterials and Devices. Vol. 7, No. 1, 2019, pp. 6-10. \\ doi: $10.11648 /$ j.nano.20190701.12
}

Received: July 19, 2019; Accepted: July 31, 2019; Published: September 2, 2019

\begin{abstract}
In this paper, ultraviolet (UV) detection properties of epitaxial $\mathrm{ZnO}$ films grown on sapphire substrates with radio-frequency magnetron sputtering is reported. The responsivity $(\mathrm{R})$ of the $\mathrm{ZnO}$ photoconductors measured at $325 \mathrm{~nm}$ wavelength shows a strong dependence on the incident power $(\mathrm{P})$, i.e., $\mathrm{R} \propto \mathrm{P}^{-\mathrm{k}}(\mathrm{k}=0.7)$. A responsivity of $3500 \mathrm{~A} / \mathrm{W}$ (or a gain of 6000) is obtained at the optical power density of $10^{-7} \mathrm{~W} / \mathrm{cm}^{2}$, and the value decreases to $1 \mathrm{~A} / \mathrm{W}$ at $10^{-2} \mathrm{~W} / \mathrm{cm}^{2}$. The ultra-high responsivity at the low power regime suggests a long lifetime (order of $10 \mu \mathrm{s}$ ) of carriers. The enhanced carrier lifetime is explained by the preferential capture of holes at the recombination centers that exist in the depletion region around grain boundaries and film surface, and the subsequent separation of photogenerated carriers. The strong dependence of the responsivity on the incident power is attributed to the modulation of space charge region width by the photogenerated holes and the resulting change in carrier lifetime.
\end{abstract}

Keywords: ZnO, High Responsivity, Epitaxial, UV, Photoconductor, GaN

\section{Introduction}

$\mathrm{ZnO}$ possesses many interesting properties and has been extensively studied for various applications such as varistors [1], transducers [2], transparent conducting electrodes [3], sensors [4], and catalysts [5]. Being wide bandgap semiconductor, single-crystalline $\mathrm{ZnO}$ films and related heterostructures have recently been drawing strong attention for their potential applications in light emitting or detecting devices in the ultraviolet (UV) range [5, 6]. $\mathrm{ZnO}$ is isomorphic to wurtzite $\mathrm{GaN}$ with good lattice match, and therefore has also been used as a buffer layer in growing high quality GaN films (or vice versa $\mathrm{ZnO}$ growth on $\mathrm{GaN}$ ) [7, 8]. Despite the isomorphic nature of the crystal structure, $\mathrm{ZnO}$ displays some of its physical properties significantly different from those of GaN. For example, $\mathrm{ZnO}$ shows a much larger exciton binding energy than GaN [9], and the resistivity can be relatively easily changed in a broad range by controlling the non-stoichiometric defects [10]. On the other hand, the $\mathrm{ZnO}$ 's material properties potentially offer a unique device application, the research and the development of optoelectronic devices based on epitaxial $\mathrm{ZnO}$ films, in general, has been limited comparing with the GaN case. This is mainly due to the difficulty in achieving $p$-type doping and in obtaining good quality Schottky contacts on $\mathrm{ZnO}$, and also to the rather complex behavior of the material involving film surface and grain boundaries [10].

The UV photoconductors based on epitaxial $\mathrm{ZnO}$ films have been reported to show high responsivities, i.e., $400 \mathrm{~A} / \mathrm{W}$ with $\mathrm{ZnO}$ films grown by metal organic chemical vapor deposition (MOCVD) or $1200 \mathrm{~A} / \mathrm{W}$ with $\mathrm{MgZnO}$ films grown with pulsed laser deposition [11]. The responsivity (and the gain) of a photoconductor is basically determined by the ratio of the carrier lifetime to the transit time, and is, in general, known to depend on the incident power level. The dependence of the responsivity on the incident power is an important information not only for device operation but also for materials study, since it usually reflects the gain mechanisms involved, i.e., carrier trapping and recombination at the defects such as surface/interface states at the film surface and grain boundaries. In the case of epitaxial GaN films, the UV detection properties of photoconductors have been extensively studied and the gain mechanisms have been reasonably well understood [12]. In the case of epitaxial $\mathrm{ZnO}$ films, however, 
the reports are rare that dealt with the power dependence of responsivity and the gain mechanisms responsible for the high responsivities observed. In this letter, the ultraviolet detection properties of epitaxial $\mathrm{ZnO}$ films grown by $\mathrm{rf}$ magnetron sputtering is reported. Ultra-high responsivity of $3000 \mathrm{~A} / \mathrm{W}$ is obtained with the $\mathrm{ZnO}$ photoconductors. The power dependence of the responsivity was characterized over five decades of incident power, and the result is explained with a gain mechanism that involves interactions of photogenerated carriers with the recombination centers at grain boundaries and film surface.

\section{Experimental Work and Results}

The $\mathrm{ZnO}$ epitaxial films used in this study were grown on $\mathrm{Al}_{2} \mathrm{O}_{3}$ substrates using RF sputtering. A 5N-purity $\mathrm{ZnO}$ target was used in conjunction with $\mathrm{Ar}$ or $\mathrm{Ar} / \mathrm{O}_{2}$ gas as an ambient gas for sputtering. The films were deposited at $700^{\circ} \mathrm{C}$ in Argon gas ambient. Figure 1 shows the X-ray diffraction (XRD) analysis of the $\mathrm{ZnO}$ film. The results show that the films are highly epitaxial with (0001) orientation as shown. Both the electrical properties of the $\mathrm{ZnO}$ films and the contact properties of aluminum on $\mathrm{ZnO}$ were characterized with a transfer length measurement (TLM) method. 150-nm-thick Al electrodes were deposited on $\mathrm{ZnO}$ using thermal evaporation in conjunction with a liftoff process. Aluminum source was used for the evaporation process. In order to investigate the effect of annealing on film and contact properties, some samples were further processed with an annealing at $800^{\circ} \mathrm{C}$ in oxygen ambient for $30 \mathrm{~min}$ prior to the contact metallization $[14,15]$.

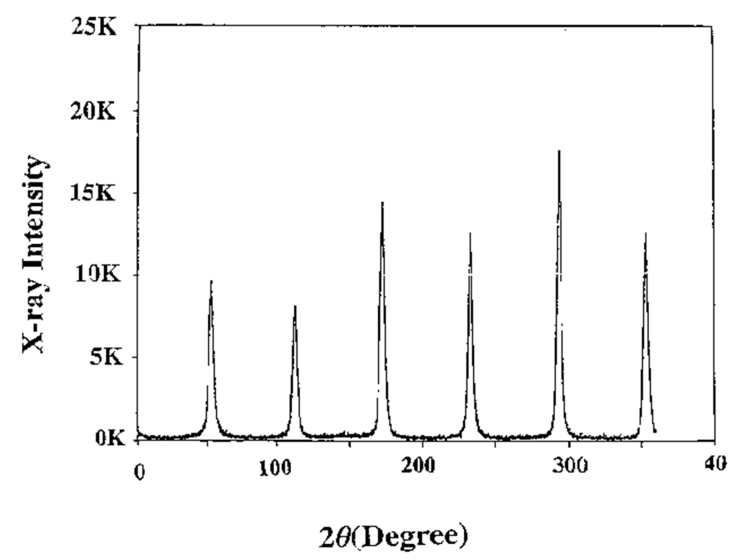

Figure 1. X-ray diffraction analysis $\theta / 2 \theta$ scan profile of $\mathrm{ZnO}$ film grown on a sapphire $[14,15]$.

Figure 2 shows the current-versus-voltage (I-V) characteristic of an as-deposited sample. It shows a linear behavior indicating the ohmic nature of the Al contact on $\mathrm{ZnO}$. Figure 3 shows the resistance values measured as a function of inter-electrode spacing. The external voltage applied across two electrodes can be divided into two components: 1) the voltage drop that appears across the $\mathrm{ZnO}$ spacing region in between the two electrodes and 2) the voltage drops in the contact region. From the slope of the I-V curve, the resistivity of our $\mathrm{ZnO}$ film ( $3 \mu \mathrm{m}$ thick) is calculated to be $200 \mathrm{k} \Omega-\mathrm{cm}$. Assuming the electron mobility of $10 \mathrm{~cm}^{2} / \mathrm{V}$-sec, the carrier concentration is estimated to be $3 \times 10^{12} \mathrm{~cm}^{-3}$. The contact resistance $\left(R_{c}\right)$ is read to be $1 \mathrm{M} \Omega$ from the $y$-intercept, and the transfer length $\left(\mathrm{L}_{\mathrm{T}}\right)$ of $1 \mu \mathrm{m}$ from the $\mathrm{x}$-intercept. The specific contact resistivity $\left(\rho_{\mathrm{c}}\right)$ is then calculated to be $5 \Omega-\mathrm{cm}^{2}$ using the relationship of $\rho_{c}=R_{c} W L_{T}$, where contact width $W$ of $500 \mu \mathrm{m}$ was used in this TLM work. This contact resistivity value is considered very large, at least three or four orders of magnitude higher than those that can be obtained with other semiconductors such as $\mathrm{Si}, \mathrm{GaAs}$ and $\mathrm{GaN}$. It is interesting to note that the $\mathrm{Al}$ contact on $\mathrm{ZnO}$ shows an ohmic character despite the relatively large value of the specific contact resistivity. With the help of figure 3 it can be shown that the amount of the voltage drop in the contact region would be equal to the drop across the spacing region of a film if the electrode spacing is designed to be equal to the transfer length. In the case that the electrode spacing is 10-20 $\mu \mathrm{m}$ and the transfer length is $1 \mu \mathrm{m}$, the voltage drop in the contact region would account for $5-10 \%$ of the external voltage applied $[14,15]$.

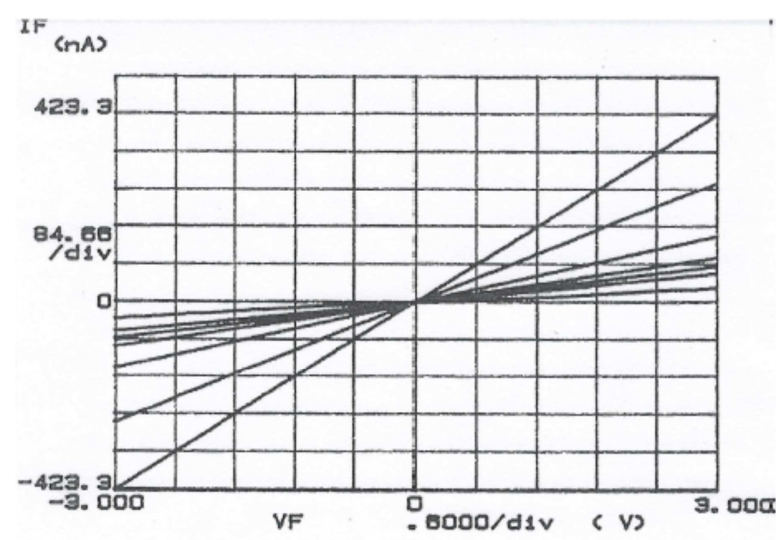

Figure 2. I-V characteristic of an as-deposited ZnO film [14, 15].

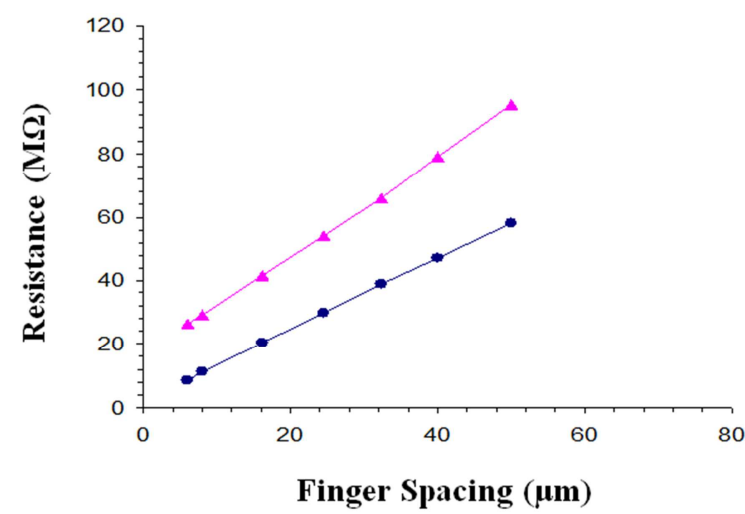

Figure 3. Resistance values measured at different electrode spacing [14, 15].

Figure 4 shows the I-V characteristics of a sample that was annealed at $800^{\circ} \mathrm{C}$ in oxygen ambient for 30 min prior to the aluminum metallization $[14,15]$. It shows an ohmic behavior similar to the unannealed sample case. Figure 5 shows the resistance values measured at different electrode spacing. The $\mathrm{ZnO}$ film resistivity is calculated to be $400 \mathrm{k} \Omega-\mathrm{cm}$. The contact resistance and the transfer length are read to be $8 \mathrm{M} \Omega$ 
and $6 \mu \mathrm{m}$, respectively, which correspond to the specific contact resistivity of $240 \Omega-\mathrm{cm}^{2}$. It is interesting to note that the contact resistance value increased 50 times after the annealing whereas the film resistivity increased only slightly (2 times). This suggests that the oxygen annealing affected mostly the film surface properties not the film bulk. The result may be explained with the following phenomenon that might have occurred on $\mathrm{ZnO}$ surface with the annealing process: The annealing in oxygen ambient might have increased oxygen concentration (i.e., coverage) on the $\mathrm{ZnO}$ surface. Considering the fact that formation of alumina is thermodynamically favored over $\mathrm{ZnO}$ formation (i.e., $\Delta \mathrm{H}_{\mathrm{Al} 2 \mathrm{O} 3}=-1675.7 \mathrm{~kJ} / \mathrm{mol}$ versus $\Delta \mathrm{H}_{\mathrm{ZnO}}=-350.5 \mathrm{~kJ} / \mathrm{mol}$ ), the increased oxygen concentration at the $\mathrm{Al} / \mathrm{ZnO}$ interface might have enhanced oxidation of Al. Formation of a thin insulating oxide layer in between $\mathrm{Al}$ and $\mathrm{ZnO}$ is then expected to increase the contact resistance. The oxygen annealing might also have altered the nature of surface states of $\mathrm{ZnO}$ in terms of their density and energy distributions, causing a change in the amount of Fermi level pinning and band bending and therefore the contact resistance. This surface phenomenon is expected to depend on the polarity of $\mathrm{ZnO}$ surface $[14,15]$. Detailed mechanism, however, is not known and is a subject of further study.

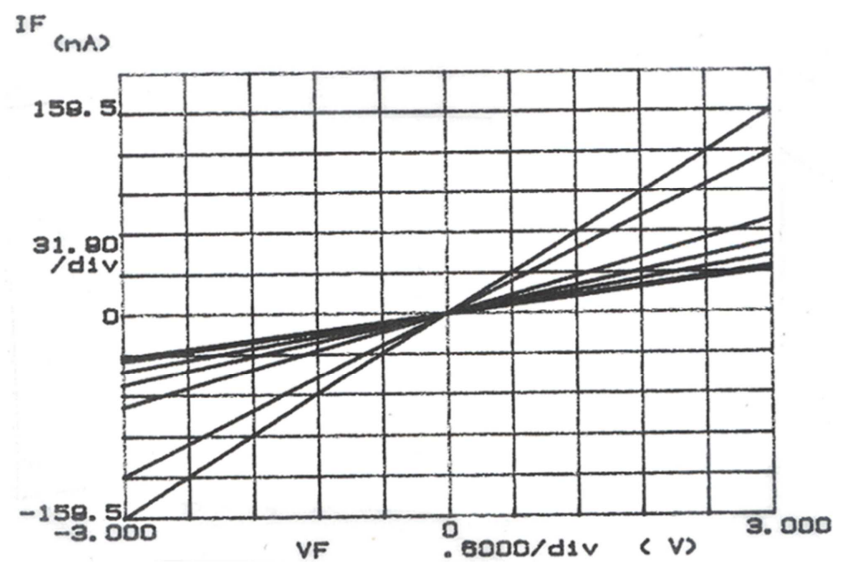

Figure 4. I-V characteristics of a sample that was annealed at $800^{\circ} \mathrm{C}[14,15]$.

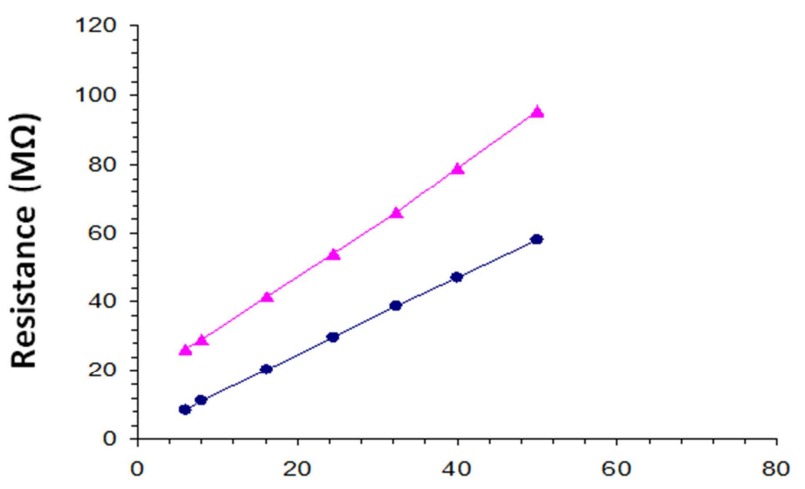

Finger Spacing $(\mu \mathrm{m})$

Figure 5. Resistance values measured at different electrode spacing $\mathbf{\Delta}$ Resistance for annealed $\mathrm{ZnO}$ films and $\bullet$ Resistance for as-deposited $\mathrm{ZnO}$ films $[14,15]$.

For UV detection experiment with a top (vertical) illumination configuration, the interdigitated aluminum electrodes (150 nm thick) were formed on both the as-grown and annealed $\mathrm{ZnO}$ samples. The electrodes were designed to have finger spacing of 10 to $20 \mu \mathrm{m}$, finger width of $10 \mu \mathrm{m}$, and finger length of $100 \mu \mathrm{m}$. The total area of the inter-electrode spacing is $2.5 \times 10^{4}{\mu \mathrm{m}^{2}}^{2}$. A $10-\mathrm{mW} \mathrm{He}-\mathrm{Cd}$ laser $(325 \mathrm{~nm}$ wavelength) was used as a UV source. The optical power density was varied in a broad range using an attenuator and also by expanding the beam. The incident power density was measured using a calibrated Si UV detector $[14,15]$.

Figure 6 and figure 7 show the photocurrent-versus-voltage characteristic of an unannealed sample measured at the illumination power level of $1 \times 10^{-2} \mathrm{~W} / \mathrm{cm}^{2}$ and $1 \times 10^{-5} \mathrm{~W} / \mathrm{cm}^{2}$, respectively. The dark current is also shown in figure 8 . All the photo and dark currents show linear dependence on the applied voltage, indicating that the $\mathrm{Al}$ contact on $\mathrm{ZnO}$ maintains an ohmic nature independent of the carrier modulation. The photocurrent is read to be $28 \mathrm{~mA}$ and $2 \mathrm{~mA}$ at $3 \mathrm{~V}$ with the power density of $1 \times 10^{-2} \mathrm{~W} / \mathrm{cm}^{2}$ and $1 \times 10^{-5} \mathrm{~W} / \mathrm{cm}^{2}$, respectively, while the dark current is read $1 \mu \mathrm{A}$ at the same voltage. This corresponds to the ON/OFF ratio of 2,000 to 28,000 in this power range. The responsivity $R$, defined as the ratio of the photocurrent over the optical power incident to detector surface, was calculated from the photocurrent measurement results. Figure 9 shows the responsivity measured as a function of the incident power density [14, 15]. The $\mathrm{ZnO}$ photoconductor shows the responsivity as high as $500 \mathrm{~A} / \mathrm{W}$ measured at $1 \times 10^{-5}$ $\mathrm{W} / \mathrm{cm}^{2}$. The photoconductor gain $(\mathrm{G})$ values are also shown in the same figure. The gain is calculated from the responsivity value using the following relationship, $\mathrm{G}=\mathrm{R} \mathrm{hv} / \mathrm{\eta q}$, where $h$ is the Planck constant, $v$ is the frequency of the UV beam $(\lambda=325$ $\mathrm{nm}), q$ is the electron charge, and $\eta$ is the quantum efficiency. $\eta$ $=1$ is assumed in this work. The gain of 20 to 2000 is obtained from the $\mathrm{ZnO}$ photoconductor. The responsivity (and the gain) shows strong dependence on the incident power, $\mathrm{R} \propto \mathrm{P}^{-\mathrm{k}}$ with $\mathrm{k}$ $=0.7[14,15]$.

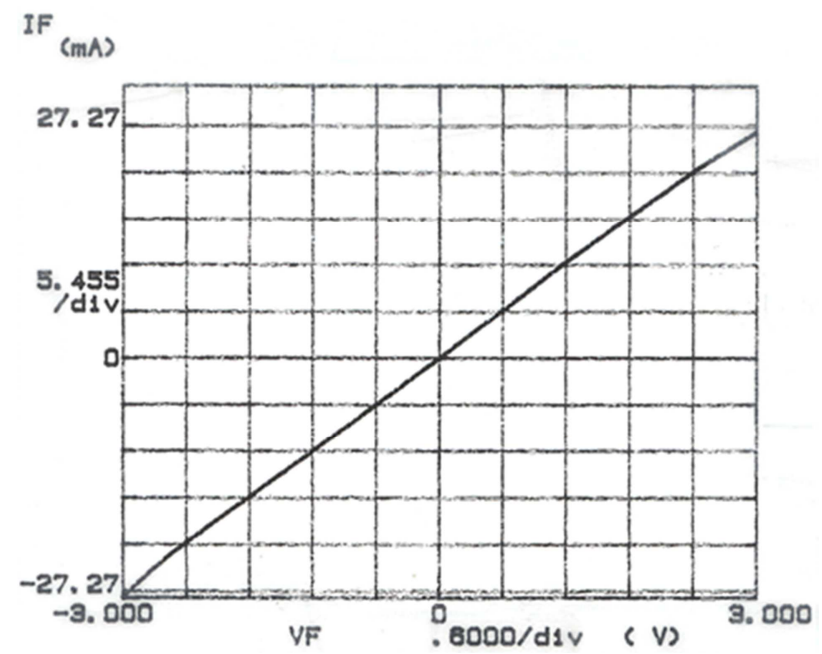

Figure 6. Photocurrent-versus-voltage characteristic of an unannealed sample with UV photoresponse at $1 \times 10^{-2} \mathrm{~W} / \mathrm{cm}^{2}[14,15]$. 
IF $(m A)$

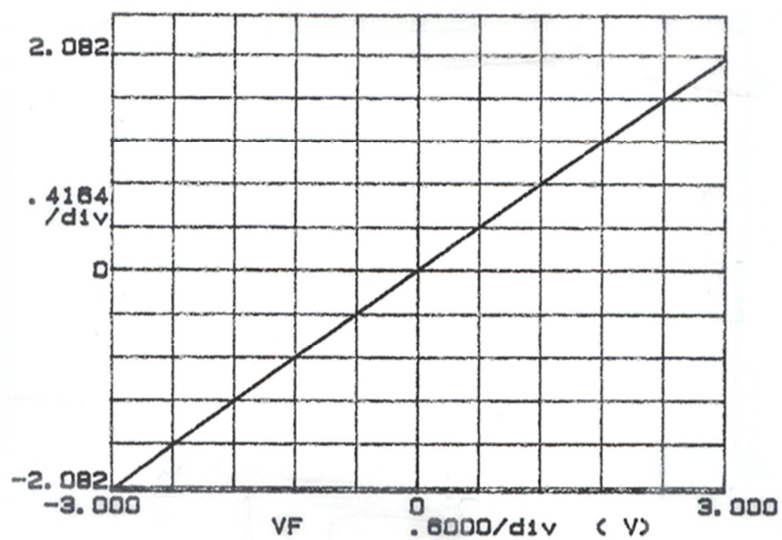

Figure 7. Photocurrent-versus-voltage characteristic of an unannealed sample with UV photoresponse at $5 \times 10^{-5} \mathrm{~W} / \mathrm{cm}^{2}[14,15]$.

IF

(UA)

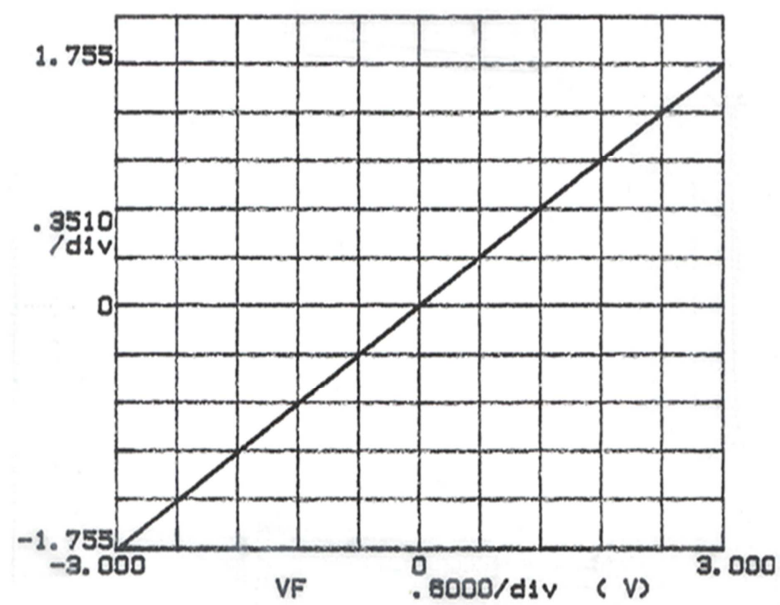

Figure 8. Photocurrent-versus-voltage characteristic of an unannealed sample for Dark current [14, 15].

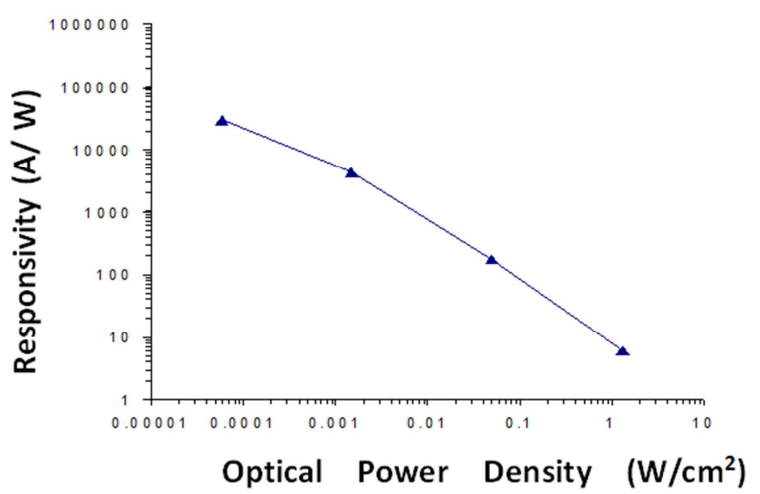

Figure 9. Responsivity vs. Optical Power Density [14, 15].

It is important to mention that a similar behavior of the power dependence of responsivity of $R \propto P^{-k}(\mathrm{k}=0.9)$, has been reported with $\mathrm{GaN}$ photoconductors $[14,15]$. Figure 10 shows the gain (and the responsivity) shows two orders of magnitude change (from 2000 to 20) as the power level increased three orders of magnitude $[14,15]$. Assuming that the conductive volume modulation is much less than the carrier modulation and also that no change in the donor concentration, the carrier mobility (and therefore the carrier transit time) is expected to maintain nearly the same level under different illumination power [14, 15]. This power dependence of gain (and the responsivity) observed in this work then suggests a strong dependence of the effective carrier lifetime on the illumination power [14, 15].

The undoped $\mathrm{ZnO}$ usually shows $n$-type conductivity mainly ascribed to the non-stoichiometric defects such as oxygen vacancies and/or $\mathrm{Zn}$ interstitials [13]. Considering the surface states on film surface and the resulting Fermi level pinning effect, a space charge region would form near the film surface and therefore a band bending occurs. Under UV illumination, the photocarriers are generated and the holes are swept to the depletion (i.e., space charge) region and captured by the surface states. This results in the reduction of space charge region width. The amount of this modulation would depend on the background carrier concentration and the illumination power. As discussed above, the modulation of charge region width itself is expected to be relatively insignificant compared with the carrier modulation effect in the $\mathrm{ZnO}$ photoconductors studied in this work [14, 15]. However, the carrier modulation would cause a certain amount of band bending change. In the dark, two opposite electron fluxes exist over the barrier, namely the thermionic emission from the metal (i.e., the aluminum electrode) towards the n-type $\mathrm{ZnO}$ and a compensating flux from the $\mathrm{ZnO}$ towards the metal. Under constant illumination, the photogenerated carriers reduce the band bending by $\mathrm{V}_{\mathrm{ph}}$, and this allows an electron flux to reach the surface equal to the holes arriving by drift and equal to the photocarriers generated $[14,15]$. Figure 10 shows the gain-versus-power curves calculated with various different barrier heights. In this calculation the transit time of $10 \mathrm{~ns}$ is used assuming an electron mobility of $10 \mathrm{~cm}^{2} / \mathrm{V}$-sec and $l=20 \mu \mathrm{m}$ and $\mathrm{V}=3 \mathrm{~V}$. The Richardson constant $\mathrm{A}=110$ was assumed in this calculation. The calculation $\Delta \psi_{o}=0.8 \mathrm{eV}$ shows a best fit with the measurement result $[14,15]$. The gain curve shows a power dependence of $\mathrm{P}^{-0.8}[14,15]$.

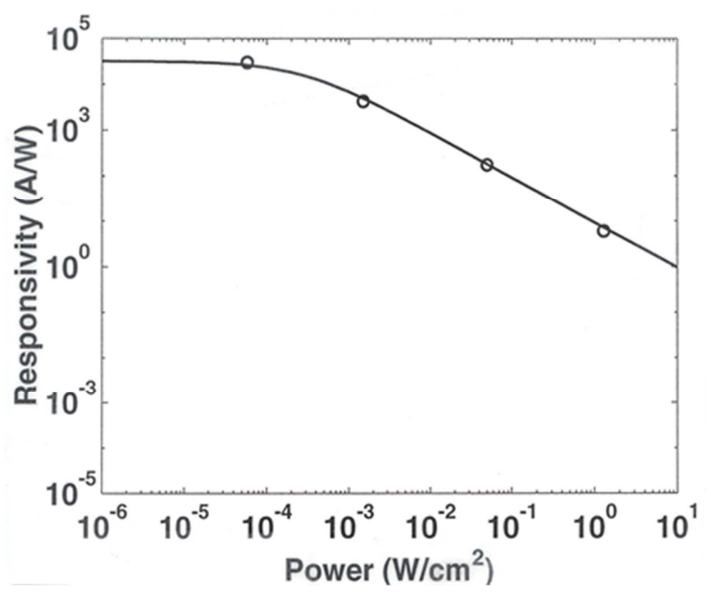

Figure 10. Gain-versus-power curves calculated with various different barrier heights [14, 15]. 
In $n$-type photoconductive devices, the gain is defined/interpreted as the number of electrons circulating through the sample per absorbed photons per unit time [14, 15]. The gain can be expressed as the ratio of the hole lifetime to the electron transit time. The annealing has an effect in reducing the surface states, reducing the lifetime enhancement. It also causes an increased in the contact resistance and reduced the voltage drop across the $\mathrm{ZnO}$ region. Similar to the GaN case, the present $\mathrm{ZnO}$ technology is far from producing low defect-density epitaxial films. $\mathrm{ZnO}$ epilayers are known to have a large concentration of dislocations, which result from the low-angle grain boundaries. It has been reported that epitaxial $\mathrm{ZnO}$ films grown on sapphire consist of columnar grains slightly twisted along the c-axis. A dislocation density of on the order of $1 \times 10^{10} \mathrm{~cm}^{-2}$ order has been reported, which corresponds to the average grain size of $0.1 \mu \mathrm{m}[14,15]$.

\section{Conclusions}

The UV detection properties of epitaxial $\mathrm{ZnO}$ films grown on sapphire substrates with radio-frequency magnetron sputtering is reported. High responsivity of $\mathrm{ZnO}$ photoconductors of $3500 \mathrm{~A} / \mathrm{W}$ (or a gain of 6000) was obtained at the optical power density of $10^{-7} \mathrm{~W} / \mathrm{cm}^{2}$, and the value decreases to $1 \mathrm{~A} / \mathrm{W}$ at $10^{-2} \mathrm{~W} / \mathrm{cm}^{2}$. The ultra-high responsivity at the low power regime suggested the long lifetime (order of $10 \mu \mathrm{s}$ ) of carriers. The enhanced carrier lifetime was explained by the preferential capture of holes at the recombination centers that exist in the depletion region around grain boundaries and film surface, and the subsequent separation of photogenerated carriers. The strong dependence of the responsivity on the incident power was attributed to the modulation of space charge region width by the photogenerated holes and the resulting change in carrier lifetime $[14,15]$.

\section{References}

[1] P Meng, S Gu, J Wang, J Hu, J He, "Improving electrical properties of multiple dopant $\mathrm{ZnO}$ varistor by doping with indium and gallium," Ceramics International 44, 1168-1171, (2018).

[2] C Fei, H Hsu, A Vafanejad, Y Li, Q Zhou, "Ultrahigh frequency $\mathrm{ZnO}$ silicon lens ultrasonic transducer for cell-size microparticle manipulation," Journal of Alloys and Compounds, 729, 556-562, (2017).

[3] B Sarma, BK. Sarma, "Role of residual stress and texture of $\mathrm{ZnO}$ nanocrystals on electro-optical properties of $\mathrm{ZnO} / \mathrm{Ag} / \mathrm{ZnO}$ multilayer transparent conductors," Journal of Alloys and Compounds 734, 210-219, (2018).

[4] M Zhang, H Zhang, L Li, K Tuokedaerhan, Z Jia, "Er-enhanced humidity sensing performance in black $\mathrm{ZnO}$-based sensor," Journal of Alloys and Compounds 744, 364-369, (2018).

[5] Q Sun, Y Men, J Wang, S Chai, Q Song, "Support effect of $\mathrm{Ag} / \mathrm{ZnO}$ catalysts for partial oxidation of methanol," Inorganic Chemistry Communications 92, 51-54, (2018).

[6] J Kim, B Kang, H Jeong, S Kim, S Kang, "Quantum dot light emitting diodes using size-controlled $\mathrm{ZnO}$ NPs," Current Applied Physics 18, 681-685, (2018).

[7] S Safa, S Mokhtari, A Khayatian, R Azimirad, "Improving ultraviolet photodetection of $\mathrm{ZnO}$ nanorods by $\mathrm{Cr}$ doped $\mathrm{ZnO}$ encapsulation process," Optics Communications 413, 131-135, (2018).

[8] D You, C Xu, F Qin, Z Zhu, W Liu. "Interface control for pure ultraviolet electroluminescence from nano $\mathrm{ZnO}$ based heterojunction devices," Science Bulletin 63, 38-45, (2018).

[9] S Park, W Hong, J Kim, "Piezoelectric and spontaneous polarization effects on exciton binding energy and light emission properties of wurtzite $\mathrm{ZnO} / \mathrm{MgO}$ quantum dots," Solid State Communications 261, 21-25, (2017).

[10] X Liang, L Shen, "Interfacial thermal and electrical transport properties of pristine and nanometer-scale $\mathrm{ZnS}$ modified grain boundary in $\mathrm{ZnO}$ polycrystals," Acta Materialia 148, 100-109, (2018).

[11] K Liu, M Sakurai, M Aono, " $\mathrm{ZnO}$ based ultraviolet photodetectors," Sensors 10, 8604-8634 (2010).

[12] L Vikas, K Vanaja, P Subha, M Jayaraj, "Fast $U V$ sensing properties of n-ZnO nanorods/p-GaN heterojunction," Sensors and Actuators A: Physical 242, 116-122, (2016).

[13] C Pemmaraju, TArcher, R Hanafin, S Sanvito, "Investigation of n-type donor defects in Co-doped $\mathrm{ZnO}$," Journal of Magnetism and Magnetic Materials 316, 85-187, (2007).

[14] Ahmed Nahhas, H Kim, J Blachere, "Epitaxial growth of $\mathrm{ZnO}$ films on Si substrates using an epitaxial GaN buffer," Applied Physics Letters 78, 1511, (2001).

[15] Ahmed Nahhas, "Study of ZnO Epitaxial growth and MSM photodetectors," University of Pittsburgh (2001). 